Article

\title{
Designing and Integrating a Digital Thread System for Customized Additive Manufacturing in Multi-Partner Kayak Production
}

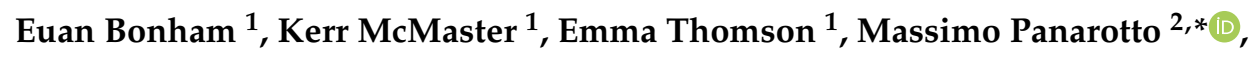 \\ Jakob Ramon Müller ${ }^{2}\left(0\right.$, Ola Isaksson ${ }^{2}\left(\mathbb{D}\right.$ and Emil Johansson ${ }^{3}$ \\ 1 Department of Mechanical and Aerospace Engineering, University of Strathclyde, 75 Montrose St, \\ Glasgow G1 1XJ, UK; euan.bonham.2015@uni.strath.ac.uk (E.B.); \\ kerr.mcmaster.2015@uni.strath.ac.uk (K.M.); emma.thomson.2015@uni.strath.ac.uk (E.T.) \\ 2 Department of Industrial and Materials Science, Chalmers University of Technology, \\ Chalmersplatsen 4, 41296 Gothenburg, Sweden; jakob.muller@chalmers.se (J.R.M.); \\ ola.isaksson@chalmers.se (O.I.) \\ 3 RISE IVF, Argongatan 30, 43122 Mölndal, Sweden; emil.johansson@ri.se \\ * Correspondence: massimo.panarotto@chalmers.se
}

Received: 27 September 2020; Accepted: 5 November 2020; Published: 10 November 2020

\begin{abstract}
Additive manufacturing (AM) opens the vision of decentralised and individualised manufacturing, as a tailored product can be manufactured in proximity to the customers with minimal physical infrastructure required. Consequently, the digital infrastructure and systems solution becomes substantially more complex. There is always a need to design the entire digital system so that different partners (or stakeholders) access correct and relevant information and even support design iterations despite the heterogenous digital environments involved. This paper describes how the design and integration of a digital thread for AM can be approached. A system supporting a digital thread for AM kayak production has been designed and integrated in collaboration with a kayak manufacturer and a professional collaborative product lifecycle management (PLM) software and service provider. From the demonstrated system functionality, three key lessons learnt are clarified: (1) The need for developing a process model of the physical and digital flow in the early stages, (2) the separation between the data to be shared and the processing of data to perform each parties' task, and (3) the development of an ad-hoc digital application for the involvement of new stakeholders in the AM digital flow, such as final users. The application of the digital thread system was demonstrated through a test of the overall concept by manufacturing a functional and individually customised kayak, printed remotely using AM (composed of a biocomposite containing $20 \%$ wood-based fibre).
\end{abstract}

Keywords: additive manufacturing; digital thread; design automation

\section{Introduction}

Compared to conventional subtractive manufacturing, additive manufacturing (AM) has numerous benefits, including minimising material waste and an increased capability to make quick and highly individualised customisations to a product [1]. Additionally, AM provides the opportunity for decentralised production, as the product can be printed wherever an AM machine is located. Manufacturing the product in close vicinity to the customer has the potential to minimise delivery time and cost, as well as reduce associated carbon emissions [2]. Such a decentralised approach to manufacturing can be enabled by digital technologies, which allow different parties to contribute to the manufacturing process. For example, a vision in the kayak manufacturing industry [3] is to allow 
users to customise their kayaks through a User Interface (UI) (Figure 1) and communicate through a central database. Such information can be used by the original equipment manufacturer (OEM) to communicate with local AM providers in the proximity of the user, who can then manufacture and deliver the kayak to the user.

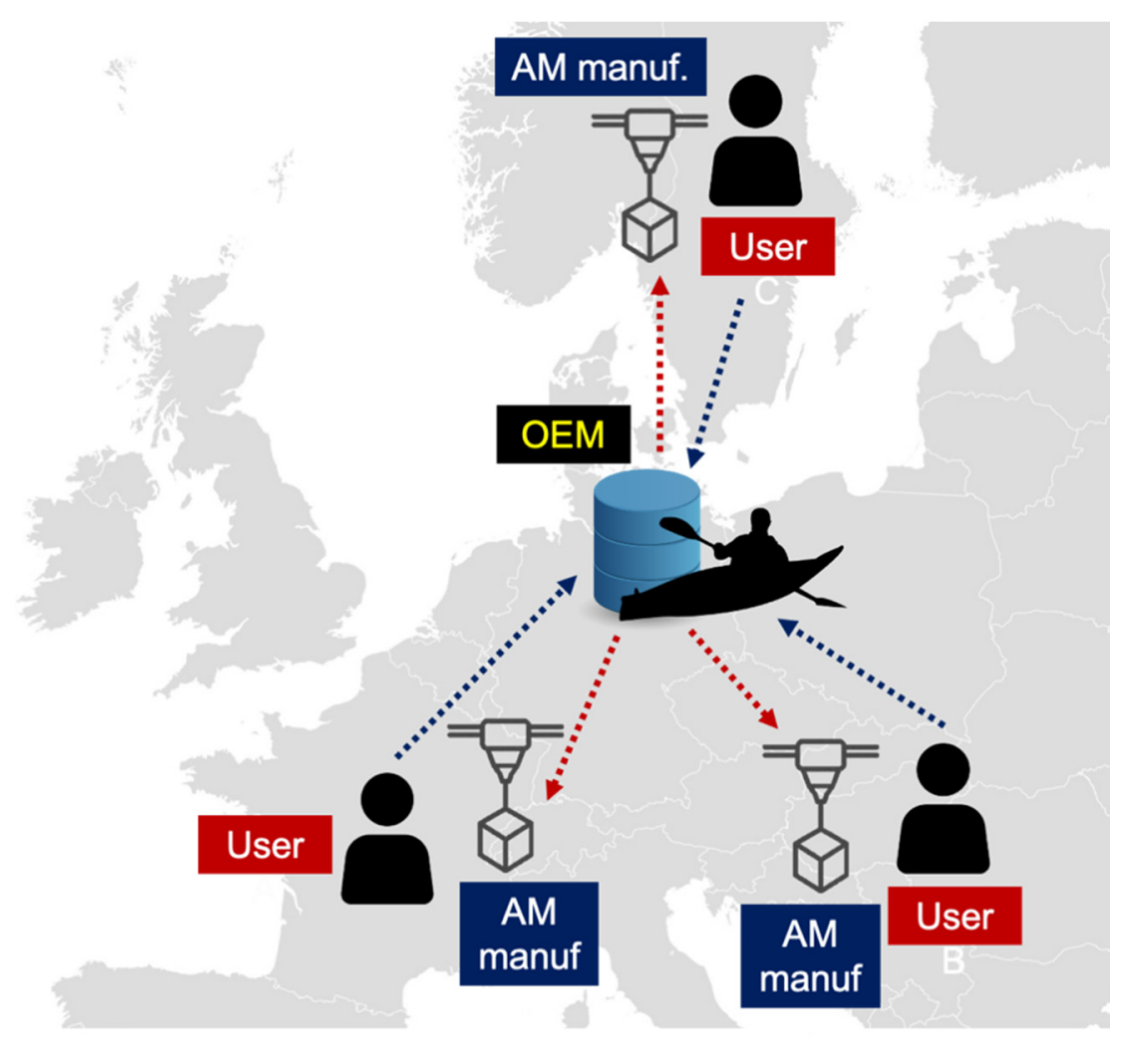

Figure 1. Vision for distributed and multi-party kayak production. The locations on the map are demonstrative only.

This approach has been covered in a previous research paper [4]; however, this previous research required significantly improved methods and multi-party integration techniques to bring it closer to commercialisation standards. These improvements are presented in this paper.

Despite the benefits of such a vision, one key challenge brought by customisation is that every individual product is traced along the supply chain due to quality assurance and warranty issues. It is important to know where and how each product has been designed. Additionally, in such a context, it is important to catalogue and trace all parties' contributions through the design and manufacturing process. For example, the transfer of production based on individual specifications from the OEM to local providers poses serious concerns for manufacturing repeatability, and it is important that faulty components can quickly be traced to source [5]. Rarely are the conditions the same for different printing locations. Another challenge is to ensure that all the partners use the most current data and can react quickly as changes are made. As in this case, the customisation procedure is an actual design step, requiring several steps to assure the validity of the specific instance being designed.

Due to the novelty of AM, there is a need to design how different parties (or stakeholders) access information and models, such that they can access appropriate information at the right time. Additionally, there is a need to design such a system in a streamlined and ideally automated way, such that it is not labour intensive. In this context, the notion of digital thread [6] is becoming increasingly important among AM manufacturers. Digital thread refers to the communication framework that allows a connected data flow and integrated view of the asset's data throughout its lifecycle across traditionally siloed perspectives from each party or stakeholder [7]. This thread also is a prerequisite to trace back the settings and conditions used throughout the design and build phase. Designing 
the digital thread for AM production is important when considering how to distinguish the different parties involved in the AM industrial system along the digital thread. This raises the following research question: how can a digital thread for AM kayak production be designed and integrated?

Therefore, this paper describes how the design and integration of a digital thread for AM can be defined in a system that allows enterprises to anticipate and communicate bi-directionally and up and downstream where the individual product is in the lifecycle. By doing this, this paper presents a digital thread system, designed and integrated for kayak AM production. The next Sections (Section 1.1, Section 1.2, and Section 1.3) provide a literature review focusing on the benefits and challenges related to AM customisation, as well as on current research in digital threads. Following this, the results of the research are shown in Section 2, detailing the main building blocks of the digital thread system for kayak production. These results are discussed in Section 3, where the implications of the presented digital thread system are discussed. Finally, Section 4 presents the research methodology that was adapted to design and integrate the digital thread system.

\subsection{Customisation in Additive Manufacturing: Potential and Challenges}

Customisation in manufacturing is motivated by the opportunity to provide users with a personalised product that more closely matches the demands of a customer compared to mass-produced products [8]. Mass customisation, as opposed to mass production, is defined by Bhrigu Ahuja et al. [9] as "the manufacture of similar but not identical products, thus enabling a unique customizable feature ideally personalized". Mass customisation originally gained popularity in the 1980 s as an opportunity for companies to gain a competitive advantage by matching demand for increasing product diversity [10]. Similarly, AM is a relatively novel field, which originally emerged to produce functional and aesthetic prototypes known as rapid prototypes [9]. Manufacturing can begin instantly once a new printable computer aided design (CAD) file is received, meaning AM offers an additional degree of geometric freedom [11], which makes it highly suitable to make quick design alterations and offer personalised products [5]. A good example is where AM is utilised to offer personalised hip and knee replacements that match a patient's geometric measurements [11].

Despite this technology's high potential and growth in the last three decades [1], the commercial uptake has been somewhat stunted for several reasons. Particularly, as the technology is evolving rapidly, the information exchange associated with the manufacturing chain has become increasingly digitalised and data-intensive [12]. To ensure the quality of the printing phase, extensive monitoring and control measurements are logged as a part of tuning and optimising the print processes. The success of the AM build process is further dependent on the design parameters and configurations made up-stream. The concept of a digital thread is a recurring theme that digitally connects all involved parties in the AM process. This is especially important when considering manufacturing and material traceability, but poses additional challenges regarding:

- Conformance with existing standards and lack of existing digital standards [13].

- The fact that manual processing of digital information can be highly labour intensive. This is made worse by a disjointed digital thread.

- Remaining adaptable to allow for new parties in the AM process (e.g., new suppliers) [14].

The research in this field identifies that to maximise the potential of AM pertaining to Mass Customisation, several digital issues must be addressed. As noted by Bonnard et al. [12,13], a key difficulty is interacting with and exchanging models, in which the AM digital supply chain convention for 3D CAD models is an STL representation. This limits the useful information that can be extracted from the model. Such digital aspects are critical when considering AM being used for customisation, as this requires local AM providers, end-users, OEMs, and designers to facilitate the digital exchange of information in a streamlined and effective way. Kim et al. [14], developed a methodology for integrating and streamlining the flow of digital information in the AM product realisation method, noting there is a need to improve how individuals can interact with data. 
As far as the authors of this paper can tell, the consideration of digital thread for AM specific to mass customisation in literature is very sparse, meaning a potential research gap exists here.

\subsection{Multi-Party Industrial Systems in Additive Manufacturing: Potential and Challenges}

Traditionally, large manufacturing businesses, such as those in aerospace, have had to deal with complex and remotely operated industrial systems. This is due to the involvement of many partners in the supply chain of an aircraft or engine. In basic terms, the supply chain can be viewed as the exchange of information, models, and materials between multiple parties in a system. The sustainability of the supply chain relies on these links being secure and traceable [15]. As the aerospace industry has grown and developed in practise to develop complex, individualised products with multi-site production, they have used their resources to create a more streamlined supply chain that relies heavily on outsourcing and collaboration. The key players in the industrial system are:

- The OEMs who are the outward-facing organisation providing the product to the customer.

- First tier suppliers who directly supply the OEM.

- Second level suppliers who are the main suppliers of the first tier.

- Third level suppliers who are the suppliers of special components or a high-skilled process.

These complex systems also have access to research and government help [16]. This has created more complex work packages and an element of risk; however, it can be designed to reduce costs and delivery times [16].

It has been said that you cannot think of a small medium enterprise (SME) as a scaled-down large business [17]. It operates differently, and an SME adopting AM can lead to a more complex industrial system than it is used to without the resources to handle it. It has been established that even in existing supply chains, information sharing is important, and when SMEs introduce AM, it will result in further multi-party collaboration where the transparency and traceability of data are essential to the success of the business and product [17]. This is an area that SMEs struggle to develop and integrate [17]. It is important that a clear solution to this problem is found to make multi-party industrial systems in AM successful. The authors have identified a gap in the literature regarding this problem in the kayak production industry and believe a solution can be found.

\subsection{Digital Threads}

To successfully upscale AM methods from basic prototyping to fully-fledged manufacturing lines, the method's core reliance on digital technologies must be addressed [18]. Whether researching new designs and experimenting with new materials or manufacturing customised components, huge quantities of data are captured throughout a product's lifecycle [19]. This data is required to optimise the manufacturing process; however, not all information should be freely available to all parties in the supply chain. Sensitive customer data should not be shared with the local manufacturers and IP must remain protected. Alongside this, the data must be stored and handled in a way that allows fault tracking back down the supply chain. This process is described through the phrase "digital thread". This is a relatively new term, and as such is not fully understood by industry yet. This provides the opportunity for research to be completed and the technology gap to be filled.

Traditionally, collection of data and commercial traceability would be achieved manually through paperwork [20]. With large projects, this method can quickly become overwhelming with strict, large data filing systems being required. In modern-day manufacturing companies, electronic software-based data recording and storage is utilised. The key advantages of AM allow the rapid creation of complex and high-value products at a low cost [21]. This process and material based optimisation generates a lot of data that requires analysing, as such this data management is one of the key issues for the commercial adoption of this technology [22]. Currently, the solution to this issue is outdated and relies on methods developed in the 1980s such as G-code and STL file type combinations [23,24], requiring several modifications and file type combinations to produce a final product. These methods require 
modernisation and a standard framework for data exchange is key to the overall contextualisation of the digital thread in this sector [25]. This standardisation will ensure key parameters will remain accessible from design to testing.

One solution could be the additive manufacturing file (AMF) that has been recently developed, which has native support for colours and materials. Even though standardising this file type would streamline the data exchange, there is still a requirement to control the movement and access rights to potentially sensitive data. However, there is little literature available on how to achieve this within this context, and as such, the technology gap remains. The standardisation of an effective digital thread remains as the primary method to close this gap.

\section{Results}

This section describes how a digital thread system for kayak production has been designed and integrated in collaboration with an established kayak manufacturer-Hereafter named as original equipment manufacturer (OEM).

\subsection{Designing a Digital Thread for Customised Additive Manufacturing in a Multi-Party Industrial System for Custom Kayaks}

The OEM has an interest in AM for its benefits of providing large scale customisable products that can be printed in proximity to the customer by local AM manufacturers. Compared to traditional manufacturing methods, AM in principle requires no pre-existing tooling; usually, only a CAD file needs to be provided for the product to be manufactured. Therefore, AM requires minimal physical infrastructure (for example, in terms of costly tooling equipment) compared to other manufacturing methods. However, one of the key challenges for the OEM in this context is that while the physical infrastructure (machinery required) appears less complex than a conventional machining process, the digital infrastructure (management of data flow) increases substantially. For distributed AM, this means sharing richer information (design and production parameters are quite dependent) effectively. The fact that the dialogue between several partners is a part of a design process means that the requirement on traceability and effectivity (actuality of data accessed) becomes decisive. For example, it is crucial that each local AM manufacturer only receives access to the required geometry files for their specific orders, and that the same information is associated with the actual state of the version communicated. This is important not just from the perspective of IP rights from the OEM, but also to avoid confusion and customer dissatisfaction through the accidental production of the wrong product. As issues with products will inevitably occur, data traceability serves an important role to isolate and track the source of error (i.e., which party supplied the failing part, etc.). To facilitate this within the digital thread, it is important to allow multi-directional data flow, but still conserve the primary objective of selective data sharing. Therefore, the first step of designing the digital thread was to create a process model of the digital thread itself, in which the physical process and the digital process are mapped alongside.

Figure 2 visualises the process model of the digital thread. This step requires the OEM to interact with the other stakeholders, as the AM physical and digital processes are substantially different compared to a process based on traditional manufacturing. Figure 2 shows the results of these interactions, using a modelling method similar to service blueprint [26]. How these interactions were conducted are described in the methodology Section 4.3. 


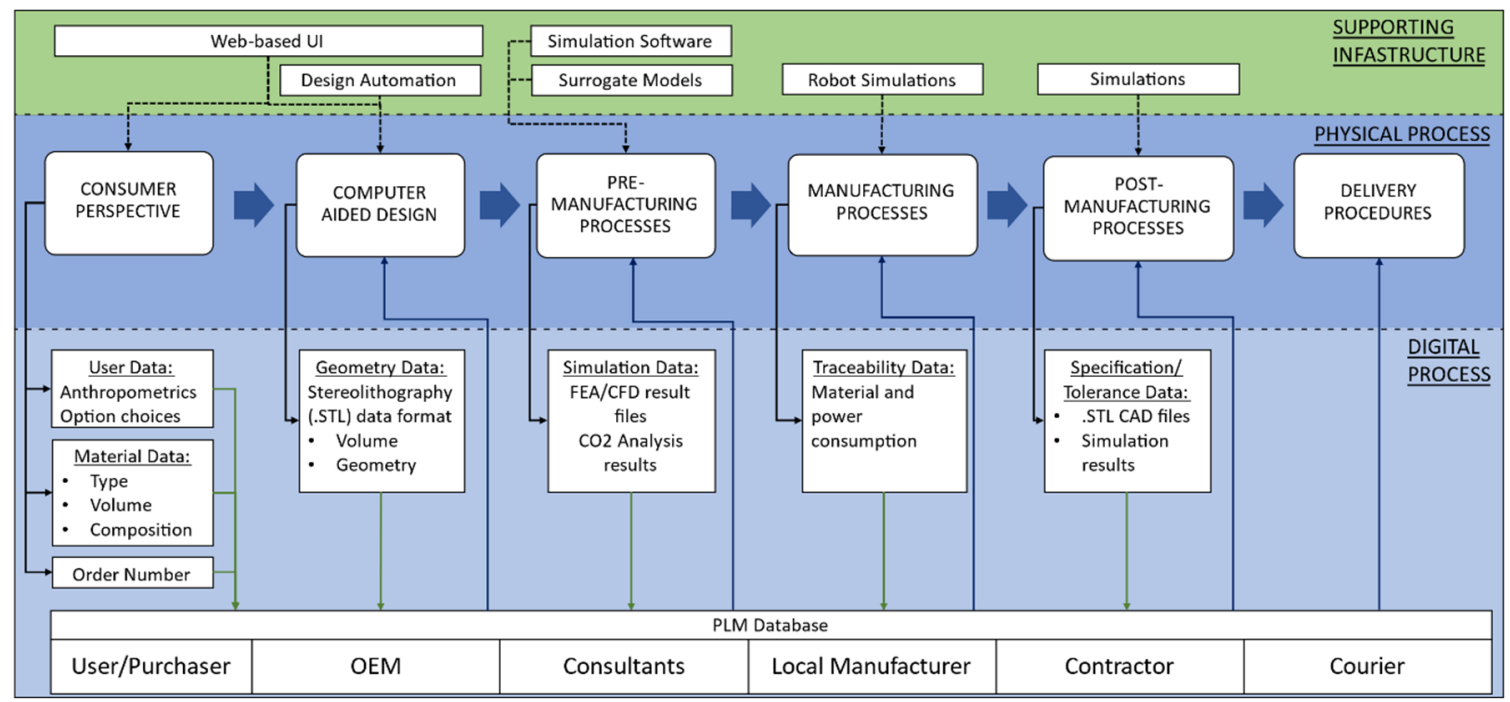

Figure 2. Process model of the digital thread for kayak AM production.

In this model, the new stakeholders that are involved in the AM kayak production are identified and mapped. For example, the OEM needs to provide the CAD model selected by the users to the local AM manufacturers. At the same time, the manufacturers must provide traceability of data such as material consumption and source, and power details for use by a $\mathrm{CO}_{2}$ consultancy firm to conduct $\mathrm{CO}_{2}$ analysis. Many manufacturers may choose to complete any post-processing required on their products inhouse; however, this may be outsourced, dependent on the specialisation required in the process (e.g., specific powder coatings or heat treating), and this will require the transfer of data regarding specification and tolerance data from the OEM together with existing measurements from the manufacturer.

\subsection{Integrating the Digital Thread}

The data generated throughout the physical process is required by various parties along with the manufacturing process flow. However, this reveals an issue with such a multi-party industrial system. Not all data should be accessed by all parties; for example, user address and card payment data require strict restrictions for both user security and data protection act compliance. Likewise, the courier does not require access to the manufacturing options such as material data that are selected at the same stage. To solve this issue, the approach taken in collaboration with a professional product lifecycle management (PLM) company was to design a digital infrastructure in which each party (or stakeholder) uses dedicated digital applications to interact bi-directionally with a single collaborative PLM platform through application programming interfaces (APIs). The motivation for this approach is to separate the information contained in the PLM platform from the data processing that each party performs to fulfil their tasks. In this way, each party can extract only the allowed information, process this data through its dedicated applications, and input this data in the PLM database. This ensures the use of the most current data at each step of the physical process, as parties working with downstream processes cannot modify the data produced upstream. Additionally, this approach allows each party to select the access rights to be allowed to other parties (e.g., only read, read/write etc.). Figure 2 visualises this separation between the collaborative PLM database and the digital applications used by each party. While some of these applications are commercial software used already by the parties (e.g., a commercial robot simulation software), some other applications needed to be developed ad-hoc to demonstrate the digital thread.

The following sections will describe in more detail these applications, in particular:

- A web-based user interface (UI) allowing kayak customers to configure their desired kayaks,

- a CAD design automation framework, to pre-generate desired kayak configurations, 
- a cost and $\mathrm{CO}_{2}$ model to visualise the cost and $\mathrm{CO}_{2}$ impacts of the selected kayaks.

The following sections will describe in more detail these applications, and how each step of the AM physical process is supported by the digital thread.

\subsection{Web-Based User Interface (UI) to Support the Customer Perspective}

The customer perspective encompasses the user and purchaser (these may be separate parties, e.g., when purchasing a product as a gift). Customers and purchasers use a web-based UI to input their user data. User data is a broad term that encompasses shipping and payment details such as user address, card numbers, delivery type, and customisation and manufacturing specific detail. Each user order is also assigned a unique order number. This is used at various stages in the process to connect all order-specific data. In this case study, a UI was created using Vue.js and Firebase. A snapshot of the UI can be seen in Figure 3. Thus, a digital application was created to effectively harvest the appropriate data from the user to create a kayak that met all their needs. Using the UI, the customer would go step by step to customise the kayak by selecting options from dropdown menus.

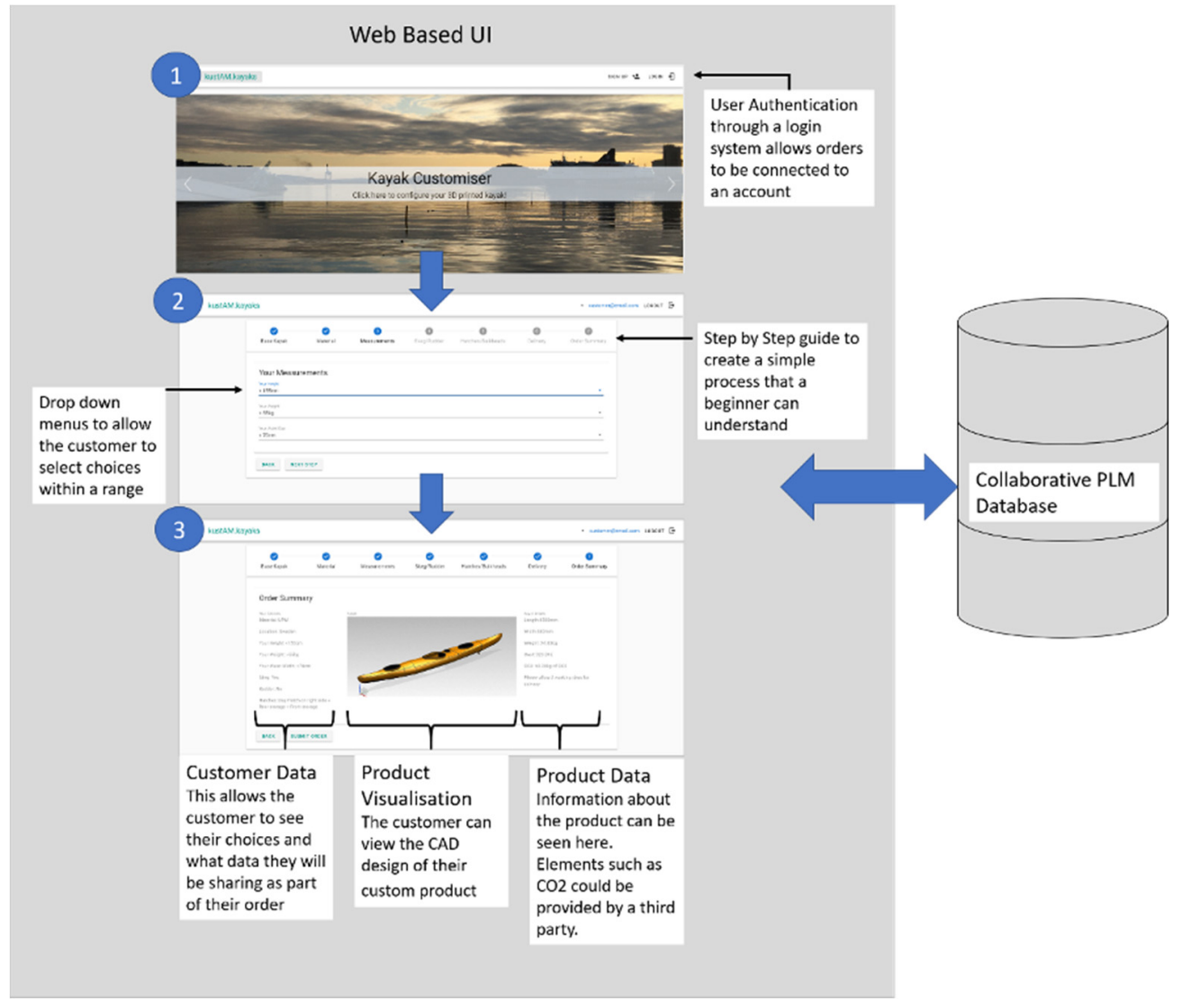

Figure 3. Web-based user interface.

These options include:

- Kayak base model,

- user anthropometrics (height, weight, and waist size),

- skeg,

- rudder,

- hatch and Storage areas,

- material selection. 
These options were identified after a workshop with the kayak OEM, focused around an exercise using a Kano model [27], to identify "attractive", "one-dimensional", and "must-be" features for a kayak. How the Kano-model was used in this research is explained in the methodology Sections 4.1 and 4.2. Once the options have been selected, the customer would be able to see a preview of their custom kayak, along with the cost and the $\mathrm{CO}_{2}$ output associated with the manufacture of their unique product.

If the customer is satisfied with the kayak, the order would be submitted to a database, where it is assigned a randomly generated order number, meaning that all data from the order can be traced to that unique key. This is important, because some of the customer's data will be shared, but ensuring the data is connected even though certain elements will be hidden from the other parties. Traditionally, this step is followed by the generation of a CAD model by a designer, who will customise the kayak based on customer preferences. As this step would be particularly labour intensive, the approach taken was to exploit design automation (DA) techniques to pre-generate all the possible kayak configurations.

\subsection{CAD-Based Design Automation Framework}

The design step in the physical process flow (Figure 2) takes the user's data, which is then fed to a Python-based journal code (Figure 4). This is a DA technique, which is based in a commercial CAD software package. A master CAD file is required to automatically be edited by this code, producing a personalised model. This will be created and operated by the OEM (i.e., the local AM manufacturers only receive a STEP file generated automatically by the OEM).

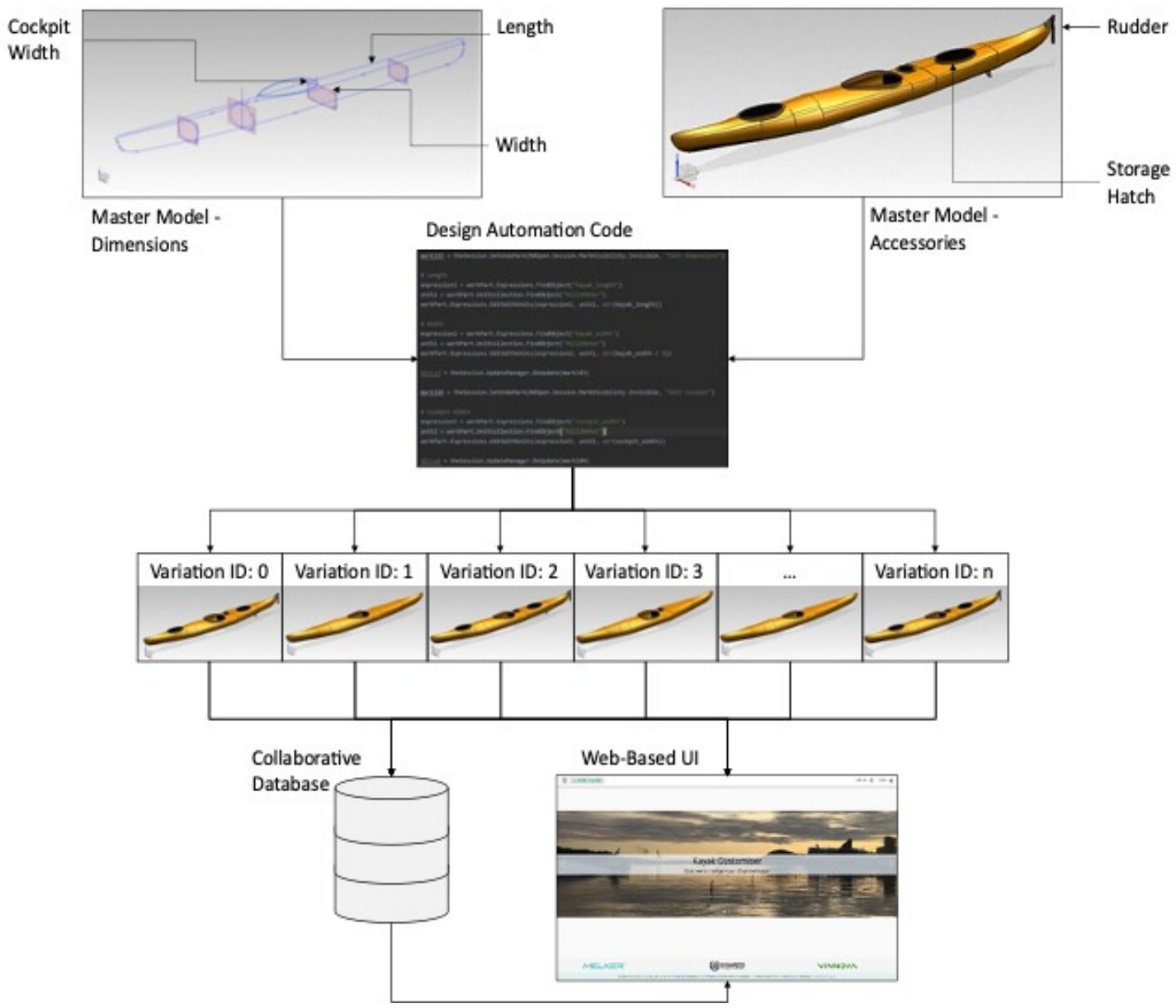

Figure 4. CAD Model Generation Sequence. 
The master CAD file is key to allowing a DA approach to CAD model generation. This required specific ratios and formula-based measurements to be input to the dimensioning and sketching phase of the CAD generation. This, combined with correct constraining of the model, allowed specific dimensions (e.g., length and width) to be input as a single dimension, and the model would update to these new specifications but maintain its overall structure and predefined shape.

This type of CAD modelling is referred to as parameterising, and the model produced is known as a master file, as when the python code is running, it selects the dimensions in the model that require changing (e.g., the cockpit width must adjust in coordination with the user's waist width) and automatically cycles each through a predefined list; examples of this can be seen in Table 1.

Table 1. CAD model variable parameters example.

\begin{tabular}{|c|c|c|c|c|c|}
\hline \multirow[b]{2}{*}{ ID } & \multicolumn{3}{|c|}{ Dimensional Parameters } & \multicolumn{2}{|c|}{ Accessories } \\
\hline & Length (mm) & Width (mm) & Cockpit Width (mm) & Storage Hatch Layout & Rudder \\
\hline 0 & 4500 & 480 & 400 & 1 & Yes \\
\hline 1 & 4500 & 480 & 400 & 1 & No \\
\hline 2 & 4500 & 480 & 400 & 2 & Yes \\
\hline 3 & 4500 & 480 & 400 & 2 & No \\
\hline$\ldots$ & $\ldots$ & $\ldots$ & $\ldots$ & $\ldots$ & $\ldots$ \\
\hline 1500 & 4500 & 480 & 400 & 7 & Yes \\
\hline 1501 & 4500 & 480 & 400 & 7 & No \\
\hline 1502 & 4500 & 500 & 430 & 1 & Yes \\
\hline 1503 & 4500 & 500 & 430 & 1 & No \\
\hline 1504 & 4500 & 500 & 430 & 2 & Yes \\
\hline 1505 & 4500 & 500 & 430 & 2 & No \\
\hline$\ldots$ & $\ldots$ & $\ldots$ & $\ldots$ & $\ldots$ & $\ldots$ \\
\hline 3195 & 5500 & 600 & 490 & 7 & Yes \\
\hline 3196 & 5500 & 600 & 490 & 7 & No \\
\hline
\end{tabular}

The code was created in a way that allowed each possible combination of both dimensional changes and the addition or absence of any optional features to be created and saved with a specified naming mechanism. This allowed thousands of CAD models to be created and stored logically without any additional human interaction or additional hours being spent. The file type can also be specified, which is important for the consistency of the digital thread and utilisation of other parties further down the process flow. Pre-generating the individual versions also allow the designer the ability to eliminate undesirable or unwanted instances, but this was not further explored in this study.

\subsection{Pre-Manufacturing Processes and $\mathrm{CO}_{2} \mathcal{E}$ Cost Model Generation}

The design of this master CAD file must be verified through an iterative collaboration with pre-manufacturing simulations, most likely outsourced to a specialised consultancy company (for complex processes, multiple external consultancies may be used). This requires geometry data output as a stereolithography (STL) file, alongside other specific requirement data, to be exchanged between the OEM and consultancy parties. From a data management perspective, this enforces the need to capture the changes and additions made in each step against the versions initiated. This stage of the process also requires a $\mathrm{CO}_{2}$ model to provide meaningful feedback for increasingly environmentally aware customers. Most likely, this model is developed by an external consultancy firm. In this case study, this was developed in house for demonstration purposes, and integrated with the UI. A life cycle assessment (LCA) approach was developed using open-source software, open LCA [28], considering only the manufacturing elements of development (i.e., no waste considerations). Figure 5 illustrates the overall logic of the developed LCA and cost models. 


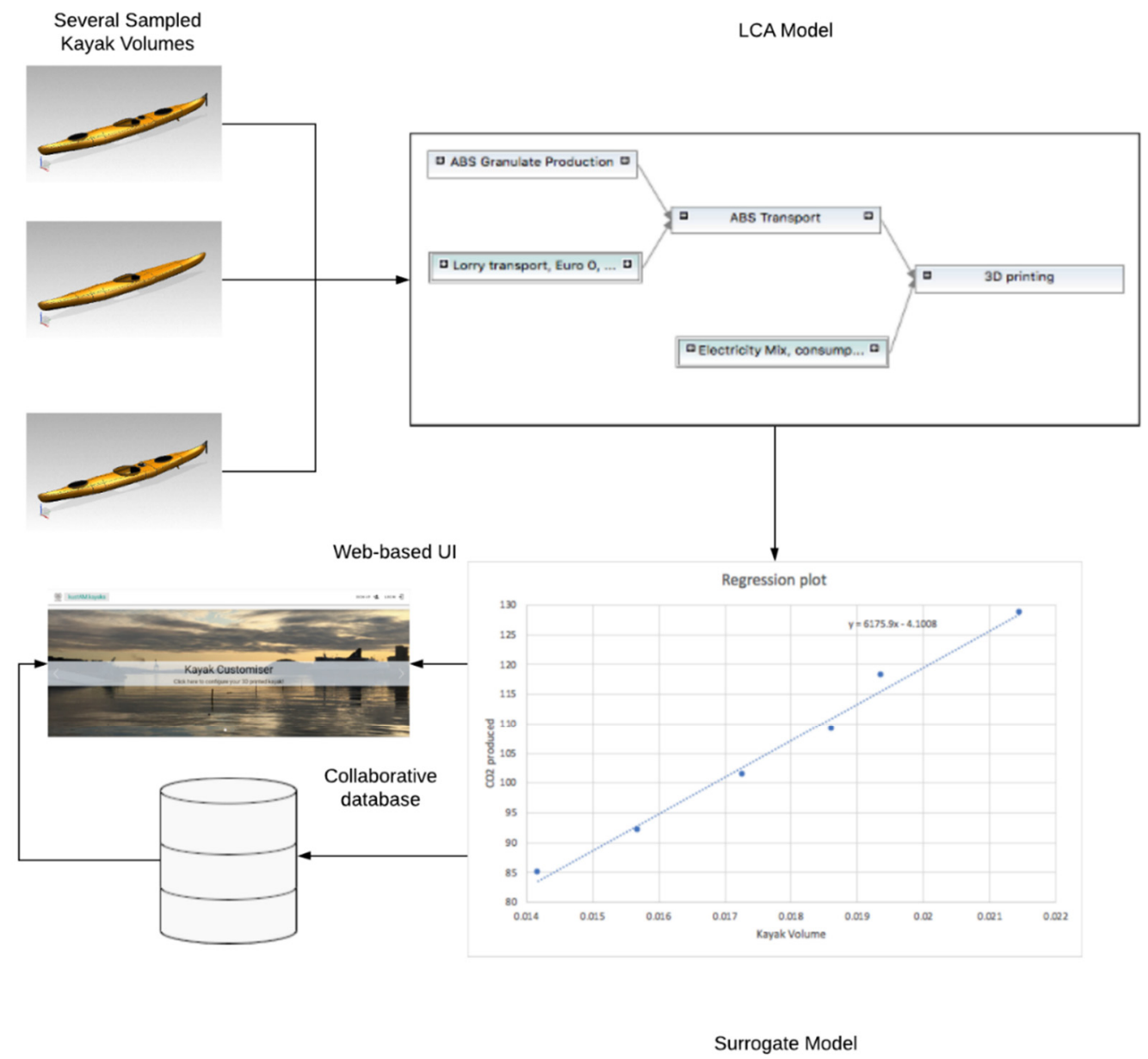

Figure 5. $\mathrm{CO}_{2}$ model visualisation.

This model pieced together the various stages of manufacture that contributed to $\mathrm{CO}_{2}$ emissions. This includes material granulate production, transportation, and electricity consumption. Figure 5 displays the role of a $\mathrm{CO}_{2}$ consultancy in this case, and how the data generated fits back into the overall process. To generate a linear prediction expression across the entire range of kayak model volumes, statistical analysis software was used to develop a surrogate linear regression model. This was achieved by sampling the volumes of several kayaks in the LCA study and then populating the $\mathrm{CO}_{2}$ emission values for intermittent kayak volumes through the model. Then the output of this model is fed back in real-time to the UI to display to the user. This information about the model and user are utilised in JavaScript Object Notation (JSON) format by the OEM for use in populating a database which feeds the web-based UI. At this stage, the purchaser will confirm their order and the STL file will be sent to the manufacturer.

Depending on the material options selected by the user and ranging kayak volumes available, the $\mathrm{CO}_{2}$ emissions varied between 41.60 and $128.51 \mathrm{~kg} \mathrm{CO}_{2}$ equivalent according to the global warming potential (GWP) as determined within the LCA software.

Similarly, a cost analysis was also important to allow the customer to be able to assess the trade-off between $\mathrm{CO}_{2}$ emissions and cost of the kayak (i.e., the customer may be willing to choose a more sustainable material at greater personal expense). The cost model considers the manufacturing costs associated with the three critical stages of the AM process, which include pre-processing, printing, and post-processing. These costs are based on the time and operator costs for each phase, as well as material costs for printing. The pre- and post-processing costs remain fixed for all kayak volumes, while the printing cost varies depending on the volume of kayak and material choice as selected by the user. At this point, the live digital input is required in a loop, as print time varies depending 
on the kayak volume. The AM provider can perform simulations to determine the print time for a corresponding geometry. For this case, this information was determined manually for a select few kayak volumes in collaboration with the AM provider, but further work is required to fully integrate such aspects digitally.

Depending on the material options, ranging kayak volumes available, and delivery location, the cost varied between $€ 746.76$ and $€ 793.61$.

\subsection{Manufacturing, Post-Manufacturing, and Delivery}

Another important step during pre-manufacturing is to conduct a robot simulation to define the most effective robot path. In the demonstration of the digital thread, robot simulations have been performed by accessing the CAD files through the collaborative PLM (Figure 6a).

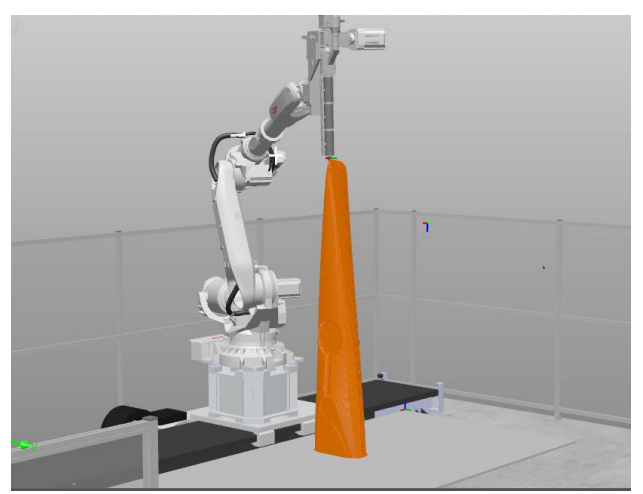

(a)

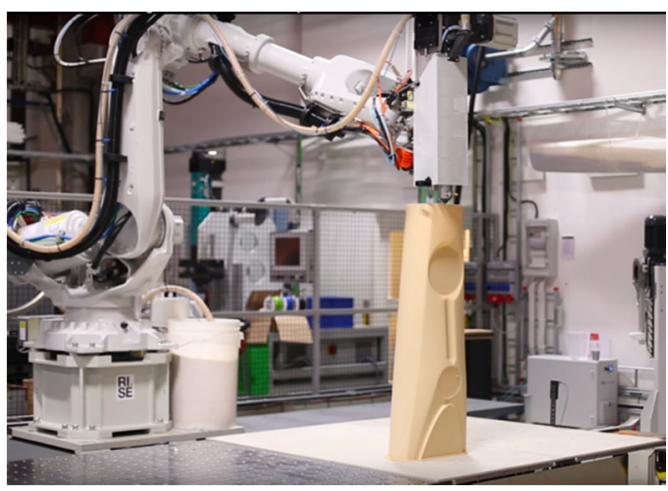

(b)

Figure 6. Remote pre-manufacturing and manufacturing of kayaks: (a) Simulation of manufacturing using ABB Robot Studio ${ }^{\circledR}$; (b) manufacturing of kayak (made of biocomposite containing 20\% wood-based fibre).

After simulations are performed (checking the feasibility of production), the manufacturing phase is executed. To demonstrate the feasibility of the digital thread system for remote production, the manufacturing was performed by a research institute that possesses dedicated equipment for large scale AM (Figure 6b). The institute remotely accessed the STL files of the kayak through the collaborative PLM interface. A functional AM kayak (composed of a biocomposite containing $20 \%$ wood-based fibre) was then manufactured.

In a real-life scenario, the completed product will require delivery to the user. Although the concept of a local manufacturer facilitates user collection of products, this may not always be feasible, and as such, a courier will be required. This party requires no information about the manufacturing process, only requiring specific user data such as address and name.

\section{Discussion}

The concept of the digital thread for AM is still evolving. Hence, the practices for designing and integrating a digital thread for AM needs to be investigated through multiple use cases with industrial partners. One such example, presented by Mandolla et al. [29], uses blockchain to support a digital twin for AM in the aircraft industry, showing great potential concerning manufacturing infrastructure and product component history. Similarly, Lui et al. [30], develops a data management system for metal AM. This effectively tackles the challenges associated with metal AM (e.g., process repeatability etc.) to manage the various product lifecycle stages and support a more efficient application of metal AM. Given the range of challenges and novel proposals in the field as well as the rapidly changing nature of the technology, it is clear there is a lack of a widely accepted framework for the digital thread for AM. There may indeed be no ideal one size fits all approach. 
Compared to previous studies, this research has put a stronger emphasis on the design aspects of a digital thread. One of the key insights for this design focus is that while with AM the physical infrastructure decreases compared to traditional manufacturing methods (e.g., less tooling equipment), the digital infrastructure increases substantially in complexity. In this regard, the model of the physical and digital flow developed in the early stages (Figure 2) shows this increase in digital complexity. Additionally, the digital applications that needed to be developed in this case study (e.g., the web-based UI and the DA framework) show how new stakeholders need to be involved in the AM digital flow in a different way than before. The results of these studies suggest to decision- and policymakers how the design of the digital interactions between the partners is decisive for the uptake of decentralised, customised, and near net shape manufacturing.

This research underlines the importance of careful management of information within AM for it to successfully integrate into the manufacturing industry [31]. This research has looked at these aspects in collaboration with a professional PLM platform provider. The industrial implementation of PLM platforms indicates its usefulness. However, examples of integrating a streamlined AM process with a corresponding digital thread using PLM in literature are scarce [32]. Indeed, Hedberg et al. [33] explore a novel PLM concept, observing that a key challenge of PLM relates to the setting in which data is required (i.e., different users along the digital thread interact with the same data in different ways, requiring different data formats, access rights, etc.). The accessibility and adaptability of such platforms is a key step towards the industrialisation of AM. One key insight that emerges from the study presented in this paper is the separation between the data to be shared and the processing of data to perform each party's task. In this way, each party can extract only the allowed information, process this data through its dedicated applications, and input this data in the PLM database. This ensures the use of the most current data at each step of the physical process.

There are limitations in this approach that need further attention in the future. Although the development of a UI to support customisable AM products through DA is not novel, it is important to remark that this was developed to link not only between the designer, manufacturer, and customer, but also to placeholder consultancies that developed and integrated cost and $\mathrm{CO}_{2}$ models associated with the AM process, enabling live feedback to be provided to the customer. The database behind the UI stored all kayak design variants, while also providing appropriate movement and access to information for the multiple parties associated with the process.

It is important to note that the digital thread that has been designed serves as a demonstration of certain capabilities, with not all parts yet fully complete. One key limitation of this approach is the requirement to redesign the digital thread for different AM applications with changing parties, with this approach being very specific to the kayak manufacture case study. Future research work will focus on the streamlined integration of the different digital tools with the collaborative platform. This would allow better traceability and data security, as it follows industry standards.

\section{Materials and Methods}

The framework for Action Research [34], was used in the development of the digital thread for the custom kayak case study. Action research describes a methodology in which researchers implement a theory or solution within a real-life situation, while also iteratively receiving feedback and improving performance. The research was conducted in close collaboration with an established kayak manufacturer, who has already invested in pre-production of AM kayaks. An important stage in the research was to identify design requirements and features for a kayak. This was achieved by conducting a Kano-model-based [27], survey in conjunction with a focus group with the industrial partners.

\subsection{Use of a Kano-Model-Based Survey to Identify Requirements and Features for Kayak Design}

The Kano model distinguishes between three different categories of product requirements:

- Must-be: Customers will expect these requirements and they are essentially taken for granted, resulting in extreme dissatisfaction if they are absent. 
- One-Dimensional: These result in satisfaction if present and dissatisfaction if they are not, with the level of customer satisfaction proportional to the amount to which the requirement is fulfilled.

- Attractive: These attributes are not expected by the customer, so when they are present, they are said to delight the customer, but when they are not, there is no dissatisfaction.

An online Kano survey was distributed to experienced sea touring kayakers. The survey was designed around a five-point scale, in which opinions about customisable features were investigated. A range of appropriate features was identified through a literature survey as well as consultation with experienced kayakers. Additionally, there was an open question at the end in which the user could enter any other kayak features that were not addressed within the survey. The total number of responses for the survey was 35 participants, and Figure 7 shows the results of the survey after applying the satisfaction and dissatisfaction indexes as described in [27]. The customer satisfaction (CS) coefficient is a useful analysis tool that identifies if satisfaction will be improved by fulfilling a product requirement, or if meeting this product requirement only avoids dissatisfaction.



Figure 7. Results of the Kano-model-based survey.

Thigh braces have a dissatisfaction coefficient of 0.79 , which leads to greater dissatisfaction if absent than satisfaction if they are present. This means that thigh braces are a product feature for kayakers that most users will be expecting (must-be). The satisfaction coefficient for the presence of a skeg was higher than the dissatisfaction coefficient, meaning this feature has more of a tendency to be an exciting or surprising feature. For internal storage, the satisfaction and dissatisfaction coefficients are similar, which is inherently characteristic of a one-dimensional feature. These results reinforce the range of importance that various features have to kayakers, indicating the suitability of kayak manufacture to providing customisable products through an AM platform.

\subsection{Focus Group to Refine the Requirements and Features for Kayak Design}

The Kano-based survey was a solid foundation for the suitability of certain product features for a customisable kayak. However, certain product features, such as internal storage, were very vague, and it was going to be a challenge to translate this directly to the modelling phase. Therefore, a focus group with the kayak manufacturer was conducted. In this focus group, the basis of the Kano model 
was explained, and then an exercise was proposed in which the kayak experts were given post-it notes. They could write down features and put them into the three categories following the Kano model. The result of this workshop is shown in Table 2.

Table 2. Kayak Features.

\begin{tabular}{cccc}
\hline Feature & Category & Configurable? & Printed? \\
\hline Deck lines & Must-be & No & No \\
Footrest & One-dimensional & No & No \\
Skeg & One-dimensional & No & Yes \\
Rudder & One-dimensional & No & Yes \\
Bulkheads & One-dimensional & No & Yes \\
Handling lugs & One-dimensional & No & No \\
Hatches & Attractive & Yes & Yes \\
Deck recesses & Attractive & Yes & Yes \\
Material choice & Attractive & Yes & Yes \\
Kayak volume & Attractive & Yes & Yes \\
Cockpit size & Attractive & Yes & Yes \\
Thigh braces & Attractive & Yes & Yes \\
Footrest position & Attractive & Yes & No \\
Personalised backrest & Attractive & Yes & No \\
\hline
\end{tabular}

One main result from the workshop was that any configurable features, that is, any features of the kayak that could be tailored to suit the anthropometric measurements or personal preference of the customer, would be attractive for the user. One-dimensional features were determined to be ones that are simply there or not, and finally, must-be features are those that are essential. Additionally, it was made apparent from the session which of the features could be 3D printed and which would be added during the post-processing phase of production.

Given that it would only be appropriate for printable features to be modelled through CAD, the session assisted with prioritising features for the kayak model. These features are shown with 'Yes' under the column 'Printed' in Table 2. Thereafter, an appropriate and verified customisation strategy had been identified to take forward into the modelling phase.

\subsection{Workshops for Designing the Digital Thread System}

The digital thread system was designed after several workshops with industrial partners. At the beginning of the study, a prototypical version of a digital thread [4], was presented to the industrial partners in training sessions led by a professional company that offers a collaborative PLM software. These training sessions defined the architecture of the digital thread and its associated digital infrastructure (and are presented in Figure 2).

Once the final design had been created, a proof of concept trial was completed. This phase of the project was to demonstrate that the company-Customer interaction tool that was developed would be a commercially appropriate option. The application of the digital thread was demonstrated through a test of the overall concept, by manufacturing a functional AM kayak (composed of a biocomposite containing $20 \%$ wood-based fibre) remotely. This proof of concept displays the feasibility of the approach developed, with multiple parties contributing to an AM process in a streamlined and effective way.

\section{Conclusions}

This paper has presented a case study aiming at designing and integrating a digital thread system for AM kayak production in which kayaks are customised and manufactured in the proximity of the customer. In this context, the tracing of sources and decisions made are key to realising a sufficient degree of quality in a customisation system (digital thread). 
Regarding the research question: how can a digital thread for AM kayak production be designed and integrated? This study has shown that designing a digital thread system is a matter of implementing a solution within a real-life situation as a proof-of-concept, in order to iteratively receive feedback from a number of relevant stakeholders. For this reason, working with design tools such as a service blueprint and the Kano model to perform a careful design and integration of the digital thread is a decisive factor to promote customisation and near net shape manufacturing. Additionally, developing a demonstration of a digital thread system on a real case was considered a crucial factor for iteratively refining the digital thread itself. While the designed digital thread system is still at a demonstration stage and can be considered as a proof-of-concept (as there is more elaborate knowledge needed within all steps), the key take away is that building a customisation system similar to the one presented represents a source of both benefits and risks for the manufacturer. The degree of complexity of the digital infrastructure is unprecedented for small-scale manufacturers such as the kayak manufacturer that participated in this research. For this reason, there is a need to capture and manage information more carefully.

Author Contributions: Conceptualisation: E.B., K.M., and E.T.; methodology: E.B., K.M., and E.T.; formal analysis: E.B., K.M., and E.T.; writing-Original draft preparation: E.B., K.M., and E.T.; writing-Review and editing: E.B., K.M., E.T., and M.P.; supervision: M.P., E.J., and J.R.M.; project administration: M.P. and E.J.; funding acquisition: O.I. All authors have read and agreed to the published version of the manuscript.

Funding: This research was funded by VINNOVA, the Swedish Energy Agency, and Formas through the strategic innovation program Produktion2030, Reference number 2017-04776. The research was performed in the DISAM project (Digitalization of Supply Chain in Swedish Additive Manufacturing).

Conflicts of Interest: The authors declare no conflict of interest.

\section{References}

1. Holmström, J.; Partanen, J.; Tuomi, J.; Walter, M. Rapid manufacturing in the spare parts supply chain: Alternative approaches to capacity deployment. J. Manuf. Technol. Manag. 2010, 21, 687-697. [CrossRef]

2. Peels, J. Decentralized Production with 3D Printing. Available online: https://3dprint.com/174375/ decentralize-production-3d-print/\#: \{\}:text=Instead\%20of\%20one\%20central\%20factory,demand\% 20using\%203D\%20printing\%20locally.\&text=Decentralized\%20production \%20close\%20to\%20customers, make\%20their\%20manufacturing\%20more\%20agile (accessed on 10 August 2020).

3. Tanner Hartmann Incredible Prints: Can You 3D Print a Functional Kayak? Available online: https: //all3dp.com/2/incredible-prints-can-you-3d-print-a-functional-kayak/ (accessed on 25 September 2020).

4. Lithgow, D.; Morrison, C.; Pexton, G.; Panarotto, M.; Müller, J.R.; Almefelt, L.; McLaren, A. Design Automation for Customised and Large-Scale Additive Manufacturing: A Case Study on Custom Kayaks. Proc. Des. Soc. Int. Conf. Eng. Des. 2019, 1, 699-708. [CrossRef]

5. Almefelt, L. Digitalized Large Scale Additive Manufacturing (DiLAM). Available online: https://www. chalmers.se/en/projects/Pages/Digitalized-Large-Scale-Additive-Manufacturing-QDiLAMQ.aspx (accessed on 10 August 2020).

6. Digital Thread for Additive Manufacturing (DTAM). Available online: https://www2.deloitte.com/us/en/ pages/public-sector/articles/digital-thread.html (accessed on 10 August 2020).

7. Siedlak, D.J.L.; Pinon, O.J.; Schlais, P.R.; Schmidt, T.M.; Mavris, D.N. A digital thread approach to support manufacturing-influenced conceptual aircraft design. Res. Eng. Des. 2018, 29, 285-308. [CrossRef]

8. Pollard, D.; Chuo, S.; Lee, B. Strategies for Mass Customization. J. Bus. Econ. Res. 2008, 6, 7.

9. Ahuja, B.; Karg, M.; Schmidt, M. Additive Manufacturing in Production: Challenges and Opportunities; Helvajian, H., Piqué, A., Wegener, M., Gu, B., Eds.; International Society for Optics and Photonics: San Francisco, CA, USA, 2015; p. 935304.

10. Mourtzis, D.; Doukas, M. Design and Planning of Manufacturing Networks for Mass Customisation and Personalisation: Challenges and Outlook. Procedia CIRP 2014, 19, 1-13. [CrossRef] 
11. Guo, N.; Leu, M.C. Additive manufacturing: Technology, applications and research needs. Front. Mech. Eng. 2013, 8, 215-243. [CrossRef]

12. Bonnard, R.; Hascoët, J.-Y.; Mognol, P.; Zancul, E.; Alvares, A.J. Hierarchical object-oriented model (HOOM) for additive manufacturing digital thread. J. Manuf. Syst. 2019, 50, 36-52. [CrossRef]

13. Bonnard, R.; Hascoët, J.-Y.; Mognol, P.; Stroud, I. STEP-NC digital thread for additive manufacturing: Data model, implementation and validation. Int. J. Comput. Integr. Manuf. 2018, 31, 1141-1160. [CrossRef]

14. Kim, D.B.; Witherell, P.; Lipman, R.; Feng, S.C. Streamlining the additive manufacturing digital spectrum: A systems approach. Addit. Manuf. 2015, 5, 20-30. [CrossRef]

15. Bales, R.R.; Maull, R.S.; Radnor, Z. The development of supply chain management within the aerospace manufacturing sector. Supply Chain Manag. Int. J. 2004, 9, 250-255. [CrossRef]

16. Moncenco, D. Supply chain features of the aerospace industry particular case airbus and boeing. Sci. Bull. -Econ. Sci. 2015, 14, 17-25.

17. Fulton, M.; Hon, B. Managing advanced manufacturing technology (AMT) implementation in manufacturing SMEs. Int. J. Product. Perform. Manag. 2010, 59, 351-371. [CrossRef]

18. Singh, V.; Willcox, K.E. Engineering Design with Digital Thread. AIAA J. 2018, 56, 4515-4528. [CrossRef]

19. Mies, D.; Marsden, W.; Warde, S. Overview of Additive Manufacturing Informatics: "A Digital Thread". Integr. Mater. Manuf. Innov. 2016, 5, 114-142. [CrossRef]

20. Collecting Production and Process Data. Available online: http:/www.leanmanufacture.net/operations/ productiondata.aspx (accessed on 8 August 2020).

21. Attaran, M. The rise of 3-D printing: The advantages of additive manufacturing over traditional manufacturing. Bus. Horiz. 2017, 60, 677-688. [CrossRef]

22. Bonnard, R.; Hascoët, J.-Y.; Mognol, P. Data model for additive manufacturing digital thread: State of the art and perspectives. Int. J. Comput. Integr. Manuf. 2019, 32, 1170-1191. [CrossRef]

23. Kwon, S.; Monnier, L.V.; Barbau, R.; Bernstein, W.Z. Enriching standards-based digital thread by fusing as-designed and as-inspected data using knowledge graphs. Adv. Eng. Inform. 2020, 46, 101102. [CrossRef]

24. Sturm, L.D.; Williams, C.B.; Camelio, J.A.; White, J.; Parker, R. Cyber-physical vulnerabilities in additive manufacturing systems: A case study attack on the.STL file with human subjects. J. Manuf. Syst. 2017, 44, 154-164. [CrossRef]

25. Xiao, J.; Anwer, N.; Durupt, A.; Duigou, J.L.; Eynard, B. Correction to: Information exchange standards for design, tolerancing and Additive Manufacturing: A research review. Int. J. Interact. Des. Manuf. IJIDeM 2018, 12, 767-768. [CrossRef]

26. Lynn Shostack, G. How to Design a Service. Eur. J. Mark. 1982, 16, 49-63. [CrossRef]

27. Sauerwein, E.; Bailom, F.; Matzler, K.; Hinterhuber, H.H. The Kano model: How to delight your customers. In Proceedings of the Nineth International Working Seminar on Production Economics, Igls/Innsbruck, Austria, 19-23 February 1996; pp. 313-327.

28. GreenDelta, openLCA. Available online: https://www.openlca.org/ (accessed on 25 September 2020).

29. Mandolla, C.; Petruzzelli, A.M.; Percoco, G.; Urbinati, A. Building a digital twin for additive manufacturing through the exploitation of blockchain: A case analysis of the aircraft industry. Comput. Ind. 2019, 109, 134-152. [CrossRef]

30. Liu, C.; Le Roux, L.; Körner, C.; Tabaste, O.; Lacan, F.; Bigot, S. Digital Twin-enabled Collaborative Data Management for Metal Additive Manufacturing Systems. J. Manuf. Syst. 2020, S0278612520300741. [CrossRef]

31. Demartini, M.; Galluccio, F.; Mattis, P.; Abusohyon, I.; Lepratti, R.; Tonelli, F. Closed-Loop Manufacturing for Aerospace Industry: An Integrated PLM-MOM Solution to Support the Wing Box Assembly Process. In Advances in Production Management Systems towards Smart Production Management Systems; Ameri, F., Stecke, K.E., von Cieminski, G., Kiritsis, D., Eds.; IFIP Advances in Information and Communication Technology; Springer International Publishing: Cham, Switzerland, 2019; Volume 567, pp. 423-430. ISBN 978-3-030-29995-8.

32. Lu, Y.; Choi, S.; Witherell, P. Towards an Integrated Data Schema Design for Additive Manufacturing: Conceptual Modeling. In Proceedings of the Volume 1A: 35th Computers and Information in Engineering Conference; American Society of Mechanical Engineers: Boston, MA, USA, 2015; p. V01AT02A032. 
33. Hedberg, T.; Feeney, A.B.; Helu, M.; Camelio, J.A. Toward a Lifecycle Information Framework and Technology in Manufacturing. J. Comput. Inf. Sci. Eng. 2017, 17, 021010. [CrossRef] [PubMed]

34. Avison, D.E.; Lau, F.; Myers, M.D.; Nielsen, P.A. Action research. Commun. ACM 1999, 42, 94-97. [CrossRef]

Publisher's Note: MDPI stays neutral with regard to jurisdictional claims in published maps and institutional affiliations.

(C) 2020 by the authors. Licensee MDPI, Basel, Switzerland. This article is an open access article distributed under the terms and conditions of the Creative Commons Attribution (CC BY) license (http://creativecommons.org/licenses/by/4.0/). 